Macedonian Pharmaceutical Bulletin, 66 (Suppl 1) 119 - 120 (2020)

Online ISSN $1857-8969$

UDC: 615.545 .014 .2

DOI: 10.33320/maced.pharm.bull.2020.66.03.059

Short communication

\title{
Critical process parameters during semisolid manufacturing
}

\author{
Viktorija Veljanoska $^{1}$, Elena Tomovska ${ }^{2}$, Milkica Gligorova $^{3}$ \\ Alkaloid AD, Blvd. A. Makedonski 12, 1000 Skopje, Republic of North Macedonia
}

\section{Introduction}

Semisolid dosage forms are very significant part of pharmaceutical dosage forms. They are advantageous in terms of its easy application, rapid formulation, and ability to topically deliver a wide variety of drug molecules.

Topical semisolid dosage forms are normally presented in the form of creams, gels, ointments or pastes. They contain one or more active ingredients dissolved or uniformly dispersed in a suitable base and any suitable excipients such as emulsifiers, viscosity increasing agents, anti-microbial agents, antioxidants or stabilizing agents (Lachman et al., 1991). The objective of this paper is to provide a clear and depth knowledge about various methods, critical process parameters and strategies for the manufacturing processes of semisolid dosage forms.

\section{Effect of raw materials on product characteristics}

A wide range of raw materials is available for the preparation of a semisolid dosage form. The choice of adequate raw materials for a formulation development is made on the basis of the drug delivery requirements and the particular need to impart sufficient emolliency or other quasimedicinal qualities in the formulation.

Semisolid dosage forms are complex formulations having complex structural elements. Mostly they are composed of two phases, one of which is a continuous (external) phase, and the other of which is a dispersed (internal) phase. The active ingredient is often dissolved in one phase, but sometimes when the drug is not fully soluble is dispersed in one or both phases, creating a threephase system. The physical properties of the dosage form depend upon various factors, including the size of the dispersed particles, the interfacial tension between the phases, the partition coefficient of the active ingredient between the phases and the rheology of the system. These factors combine to determine the release characteristics of the drug, as well as other characteristics, just like viscosity.

\section{Critical process parameters}

There are several factors that effect on product characteristics and quality such as range of temperature during production process (heating and cooling), mixing methods and speeds, pressure and usage of additional tools such as pumps.

Temperature - Range of temperature during the process is critical for successful manufacturing. Very high values can cause chemical degradation and high concentrations of degradation products which will effect on product quality and stability. Insufficient heat can lead to batch failures. Fast cooling can result with precipitation of solubilized ingredients. This step is very critical especially during emulsification step of oil-in-water emulsion. If the temperature of the water phase is much cooler than that of the oil phase, the melted constituents of the oil phase may solidify upon introduction into the aqueous phase and never properly form the emulsion, possibly even resulting in solid matter in

\footnotetext{
*vveljanoska@alkaloid.com.mk
} 
the batch. Heating too slowly can result in poor yields from evaporative loss. Heating too rapidly may burn areas of the batch in contact with the heating surface and the risk of presence of burnt material in the batch will be high. Rapid cooling can result in precipitation or increased viscosity of the product. The successful consistency of semisolids depends from proper rates of heating and cooling (Tanesh et al., 2016).

Shearing and mixing - Second very important factor that has effect on product quality is determination of type of shear and the optimal mixing times, methods and speeds. Process like emulsification requires high shear mixing or homogenization for obtaining the desired droplet size and dispersion, but there's also processes like gel formation witch require low shear in order to achieve optimal physical characteristics (like viscosity of the product). Proper mixing speeds must be obtained for each phase (Langley et al., 2019). Optimal hydration depends on the amount of shear imparted to initially disperse the polymer into the medium. If the process involves only very low shear mixing, a polymer may never be completely dispersed and hydrated, which may result inadequate viscosity. Equipment, recirculation loop with pump, may also be used to correct uniformity without changing mixing speed or time. Proper mixing speeds for each phase at every batch is very important for achieving desired characteristics of the formulation and high quality product (Nwoko et al., 2014).

Optimizing mixing time requires identifying the minimum time required for ingredients to dissolve and the maximum mixing time before product failure. For polymeric gels over-mixing, especially high shear, can break down the polymer's structure. In emulsion formulations over-mixing may result with prematurely separation of the phases which will cost with drastic changes in viscosity (Nwoko et al., 2014).

Flow rate - Flow rates are the third very important thing during manufacturing of semisolid dosage forms. Optimizing flow rate involves determining the amount of shear or throughput needed. Water-in oil emulsions require a slower addition speed than a traditional, oil-in-water emulsion, and the flow rate in this kind of formulations must be modified appropriately. Using of pumps during the process should be with very big attention because can result with changes in product characteristics. Overhearing can occur if the formulation is pumped too quickly. If pumping is too slow, the formulation will experience extra time in an in-line homogenizer, thus also exposing the formulation to additional shear. Two processes that require experimentation to optimize flow rates are the use of a powder ejection system and an in-line homogenizer. Raw material dispersers and in-line homogenizers require proper flow rates for optimal usage. If the product is not flowing through a disperser at the proper rate, there will not be enough suction for properly incorporating the powders.

Monitoring the flow rate when using an in-line homogenizer is necessary in order to calculate the theoretical number of times the product passes through it (Nwoko et al., 2014).

\section{Conclusion}

Manufacturing process for production of semisolid dosage forms is very important step for ensuring production of high quality products which will be present on the market. The quality of the product depends from many factors but mostly from the characteristics of the raw materials, equipment and their performance and critical process parameters such as temperature, pressure/ vacuum, share speed and mixing and usage of additional parts as pumps. All these parameters should be monitored during production process in defined ranges. Only fully controlled process can result with manufacturing a high quality products according the specifications.

\section{References}

Lachman, L., Lieberman, H.A., Kanig, J.L., 1991. The theory and practice of industrial pharmacy, third ed. Varghese Pub. House, Bombay, pp. 534-563.

Langley, N., Michniak-Kohn, B., Osborne, D.W., 2019. The role of microstructure in topical drug product development. Springer Nature Switzerland AG, Cham, Switzerland.

Nwoko, V.E., 2014. Semi solid dosage forms manufacturing: tools, critical process parameters, strategies, optimization and validation. Sch. Acad. J. Pharm. 3(2), 153-161.

Tanesh, S., Langley, N., Michniak-Kohn, B., Osborne D.W., 2016. Skin cream as topical drug delivery system: a review. Journal of Pharmaceutical and Biological Sciences 4(5), 149-154. 